\title{
LOS MUSEOS PEDAGÓGICOS UNIVERSITARIOS COMO ESPACIOS DE MEMORIA Y EDUCACIÓN
}

DOI: http://dx.doi.org/10.1590/2236-3459/72218

\author{
Teresa Rabazas Romero \\ Universidad Complutense de Madrid (UCM), España \\ Sara Ramos Zamora \\ Universidad Complutense de Madrid (UCM), España
}

$\cos 80$

\begin{abstract}
Resumen
Este artículo se encuadra en la nueva tendencia historiográfica emergente sobre el patrimonio históricoeducativo y el museismo pedagógico en España. Nuestro interés se va a centrar en los museos pedagógicos universitarios españoles como espacios que permiten reconstruir la memoria educativa colectiva y constituyen escenarios dinamizadores de aprendizaje para todo tipo de públicos. Estas instituciones han sido promovidas por investigadores e historiadores de la educación, procedentes del ámbito universitario, que han dinamizado la creación de estos espacios en las universidades donde trabajan. Concretamente en este trabajo se analiza con mayor detenimiento uno de estos museos, el Museo de Historia de la Educación Manuel Bartolomé Cossío, ubicado en la Facultad de Educación de la Universidad Complutense de Madrid.

Palabras clave: museos pedagógicos universitarios, museo de historia de la educación Manuel Bartolomé Cossío, memoria, patrimonio histórico-educativo, España.
\end{abstract}

\section{OS MUSEUS UNIVERSITÁRIOS PEDAGÓGICOS COMO ESPAÇOS DE MEMÓRIA E EDUCAÇÃO}

\section{Resumo}

Este artigo se enquadra na nova tendência historiográfica emergente sobre o patrimônio histórico-educativo e os museus pedagógicos na Espanha. Nosso interesse incidirá sobre os museus pedagógicos das Universidades espanholas, como espaços que permitem reconstruir a memória coletiva educacional e prever cenários de aprendizagem dinâmicos para todos os públicos. Estas instituições são promovidas por pesquisadores e historiadores da educação das universidades, que têm impulsionado a criação destes espaços nas universidades onde trabalham. Especificamente, o presente artigo analisa mais detalhadamente um destes museus, o Museu de História de la Educación Manuel Bartolomé Cossío, localizado na Faculdade de educação da Universidad Complutense de Madrid.

Palavras-chave: museus pedagógicos universitários, museu de história da educação Manuel Bartolomé Cossío, memória, patrimônio histórico-educativo, Espanha. 


\title{
PEDAGOGICAL UNIVERSITY MUSEUMS AS SPACES OF MEMORY AND EDUCATION
}

\begin{abstract}
This paper fits in the new emergent historiographical trend concerning historical-educative patrimony and pedagogical museums in Spain. Our focus is on pedagogical museums of Spanish universities as spaces that enable to reconstruct the educational collective memory and to foresee dynamic scenarios of learning for everyone. These institutions are promoted by researchers and university historians of education, who have stimulated the creation of these spaces in the institutions where they work. Specifically, this paper analyzes in more detail one of these museums, the Manuel Bartolomé Cossío Museum of History of Education, located in the Faculty of Education at Complutense University of Madrid.

Keywords: university pedagogical museums, Manuel Bartolomé Cossío museum of history of education, memory, historical-educative patrimony, Spain.
\end{abstract}

\section{LES MUSÉES UNIVERSITAIRE PÉDAGOGIE COMME ESPACES DE MÉMOIRE ET L'ÉDUCATION}

\section{Résumé}

Cet article s'inscrit dans la nouvelle tendance historiographique émergents sur le patrimoine histoire-éducatifs et les ouvrages pédagogiques en Espagne. Notre intérêt se concentrera sur les musées pédagogiques Université espagnols comme des espaces qui permettent de reconstituer la mémoire collective de l'éducation et de fournir des scénarios d'apprentissage dynamique pour tous les types de public. Ces institutions ont été promues par les chercheurs et historiens de l'éducation, des universités, qui ont stimulé la création de ces espaces dans les universités où ils travaillent. Plus précisément dans le présent document analysé plus en détail l'un de ces musées, le Musée d'histoire de l'éducation Manuel Bartolomé Cossío, situé dans la Faculté d'éducation de l'Université Complutense de Madrid.

Mots-clés: enseignement musée, musée d'histoire de l'éducation Manuel Bartolomé Cossío, mémoire, patrimoine historie-éducatif, Espagne. 


\section{Los Museos Pedagógicos Universitarios en España}

- n los últimos treinta cinco años, el despegue del museismo pedagógico en España ha sido imparable. El origen de tal cambio y proyección es complejo y múltiple. Según Julio Ruiz Berrio (2006) varios son los factores que han propiciado tal crecimiento. En primer lugar, esta evolución debe situarse en un marco global vinculado a las nuevas y diferentes orientaciones de la Nueva Museología, como movimiento científico y técnico a nivel mundial, que ofrece una concepción del museo muy alejada del positivismo científico y social y de una jerarquización museal, en el que prima una metodología monodisciplinar y una estructura centralizada. Por contra, la Nueva Museología entiende que el museo debe ser "un lugar de encuentro y aprendizaje, que se convierte en el instrumento más apropiado para que la población descubra su identidad, la asuma responsablemente y se comprometa a desarrollarla en el futuro dentro de su propio territorio o comunidad" (HERNÁNDEZ, 2006, p. 169). En este sentido, los museos actuales no representan los patrones inmutables para la cultura, sino que a través de una estructura descentralizada, una metodología interdisciplinar y un público muy heterogéneo -no sólo la clase privilegiada-, se convierten en centros de recuperación y estudio del patrimonio cultural y natural de todos los pueblos, del patrimonio tangible e intangible (RUIZ BERRIO, 2006, p. 275), adquiriendo un carácter social al servicio de las personas. Como explica Miryam Carreño, estos nuevos museos se conciben como un instrumento de concienciación que permiten recuperar desconocimientos, olvidos, voces y silencios, y actuar como instrumento liberador y socializador. (2007, p. 110).

En segundo lugar, cabe mencionar la influencia de los nuevos planteamientos pedagógicos y teorías del aprendizaje, que defienden el aprendizaje a lo largo de toda la vida y se centran más en el que aprende y no en el que enseña. En esa dirección apuntan los cambios que han operado en los museos y en concreto en los museos de educación, que se han visto influenciados por la revolución pedagógica más preocupada por una nueva cultura del aprendizaje basada en la comprensión, el análisis crítico y la reflexión. En tercer lugar, el desarrollo tecnológico, que ha obligado a la museología actual a adaptarse a los nuevas demandas sociales y promover, inmersa en la sociedad de la información, la articulación de redes de museos que permitan llegar al gran público creando nuevas instituciones museísticas más cercanas a los centros de interpretación que favorezcan un diálogo permanente con el patrimonio. (RUIZ BERRIO, 2006, p. 278).

En último lugar, reviste especial interés cómo este despegue museístico ha estado favorecido por la nueva tendencia historiográfica emergente sobre el patrimonio histórico educativo, que tiene su origen en el giro epistemológico de la Historia de la educación hacia la historia material o nueva historia de la cultura escolar ${ }^{1}$. Esta forma de plantear la historia cultural de la educación requiere nuevos enfoques como la microhistoria, la etnografía y la hermenéutica que permitan desvelar silencios a través de la mirada microanalítica donde

\footnotetext{
${ }_{1}^{1}$ Para conocer las tendencias emergentes en el contexto actual el profesor Julio Ruiz Berrio, publicó el libro La cultura escolar en Europa. Tendencias históricas emergentes en el año 2000. Esta obra nos ofrece un panorama plural de las innovaciones más actuales en el contexto nacional y europeo, desde la perspectiva del tiempo escolar (proceso de unificación de Europa), el currículum (diferencial y de construcción de disciplinas escolares en Europa), los manuales, las nuevas fuentes para la historia de la cultura escolar (educación popular, las autobiografías, los espacios eclesiásticos) y las nuevas tendencias emergentes (análisis comparativos, la nueva historia del pensamiento pedagógico y los nuevos enfoques de la posmodernidad o Alta modernidad).
} 
todo puede ser historiado. El objeto de la historia se detiene en estudiar la vida cotidiana de las instituciones, la intrahistoria de las escuelas, que es la que en realidad interesa a los educadores y a la sociedad en general, como puede ser la reconstrucción de la experiencia escolar. La aproximación a esta nueva línea historiográfica reclama la renovación de las fuentes (ecomuseos, historia oral, relatos de vida, historia material de la enseñanza, etc.). La función del historiador de la educación se plantea como un hermeneuta que debe tratar de comprender las ideas y representaciones que pueden explicar los hechos y los objetos escolares que se examinan. Todo exige estar atentos a los próximos desarrollos de la nueva corriente y profundizar más en el análisis de los grupos que mantienen distintas actitudes y en la funcionalidad de la nueva crítica cultural, para terminar cuestionando a quién puede servir. Por tanto, los nuevos enfoques renovadores plantean, como hemos dicho antes, nuevas formas de hacer historia que conectan con la experiencia, el discurso, la cultura y la identidad. Desde esta tendencia emergente es donde debemos situar el origen de los museos pedagógicos en la actualidad.

Muy vinculado a este planteamiento historiográfico, Antonio Viñao (2010) señala como el contexto en el que nacen los museos pedagógicos representa un modo de contribuir a la memoria social, institucional e individual en el campo de la educación, y siguiendo los estudios sobre la memoria en sus diferentes perspectivas, se hace comprensible en la historiografía educativa el creciente interés por los temas relacionados con la memoria y el patrimonio material e inmaterial de la educación y de la escuela. El auge entre lo patrimonial y el museismo pedagógico radica en los usos que se realizan de dicho patrimonio. Por tanto, el patrimonio educativo presenta un valor de cambio en el mercado, hay una revalorización social del mismo. Antonio Viñao destaca diferentes usos del mismo. Desde el terapéutico, como recurso didáctico -el patrimonio ofrece posibilidades para la docencia- hasta su uso académico-científico, ligado a la investigación histórica. (2010, p. 9-10).

Desde la década de los ochenta el interés creciente por la cultura escolar ha impulsado la creación de museos pedagógicos y escolares, llegando a la cifra de quinientos museos existentes en el mundo (BLACKWELL, 1992). De los cuales, unos cuatrocientos son europeos y unos cincuenta museos son españoles. Este despertar por la museología nos lleva a precisar una tipología de museos sobre la educación que elaboró el profesor Ruiz Berrio (2003): La Escuela-Museo, que se localiza en los fondos de un colegio de comienzos del siglo $\mathrm{XX}$ y que estructura sus materiales por áreas de conocimiento; el Museo Histórico Escolar, ubicado en un edificio especialmente destinado para ese fin, que exhibe lo más característico de las grandes eras pedagógicas, con un criterio cronológico desde la Antigüedad hasta nuestros días; el Museo Pedagógico, que combina una exposición de la historia de la educación con la investigación y documentación pedagógica; y el Museo de Historia de la Educación, caracterizado por su dinamismo, pues ofrece exposiciones temporales sobre diversos temas y promueve investigaciones históricas. Este mismo autor plantea (2010) que hoy en día, en España, hay dos tipos de museos. Unos se acercan al modelo de museo pedagógico en su sentido decimonónico, y otros pueden definirse como "nuevos museos pedagógicos", pues ofrecen un programa propio del siglo $X X I$, utilizando nuevas técnicas museográficas y una orientación acorde con nuevas técnicas museológicas, basándose en nuevas concepciones sobre la educación y la historia de la educación. Este nuevo enfoque supone nuevos planteamientos, estructuras, 
actividades, y sobre todo un nuevo papel del visitante, que pasa a un primer plano y se convierte en protagonista de un espacio cultural y social. (RUIZ BERRIO, 2006, p. 272).

A esta gran heterogeneidad museística, hay que añadir la procedencia de las diversas entidades que los originan: instancias políticas, universitarias, privadas, etc. Nuestro interés se va a centrar en los museos pedagógicos universitarios españoles. Estas instituciones han sido promovidas por investigadores e historiadores de la educación, procedentes del ámbito universitario, que han dinamizado la creación de estos espacios en las universidades donde trabajan. En este contexto los museos pedagógicos universitarios se plantean como espacios abiertos y vivos capaces de favorecer el estudio, la catalogación, la investigación la conservación, la protección, el uso didáctico y la difusión del patrimonio histórico educativo. Se conciben como espacios intergeneracionales que pueden ser visitados por docentes, investigadores, estudiantes, profesionales, y el público interesado en general, contribuyendo a la participación activa de toda la sociedad. Constituyen escenarios educativos más que propicios para el desarrollo del conocimiento histórico educativo, para la reconstrucción de la cultura escolar y para la puesta en valor de la memoria de la educación (ÁLVAREZ DOMíNGUEZ; PAYÁ RICO, 2015). Como explicaba Ruiz Berrio (2006, p. 290), comentado la idea de Milena Cosseto, profesora de la Libera Università di Bolzano y miembro del Comité de expertos del Museo de la Ciudad de Bolzano (Alto Adagio),

con frecuencia los Museos europeos están ubicados en centros universitarios de investigación histórico didáctica o pedagógica, por una parte porque la historia de la instrucción o de las instituciones escolares representa un importante filón de investigación; por otra porque la misma didáctica de la historia en la escuela está favoreciendo en Europa la aproximación directa a las fuentes de la historia, a los documentos, estimulando a las nuevas generaciones a apoderarse del interior de los procesos de construcción y reconstrucción de la historia". (COSSETO, 2002, p. 4).

En España contamos con una red museística de 9 museos pedagógicos universitarios:

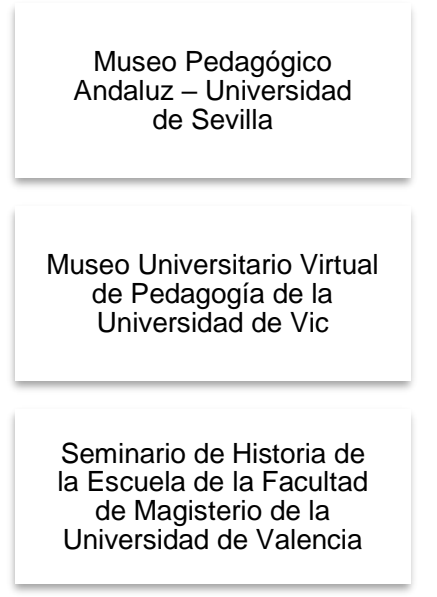

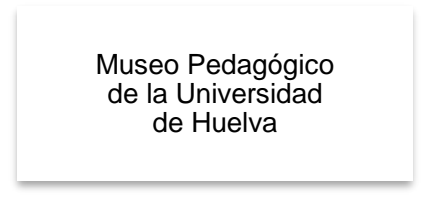

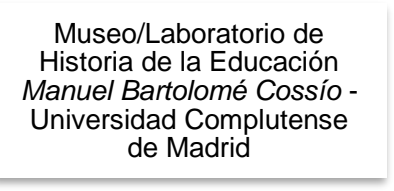

Museo de la Edudación de la Universidad de La Laguna (MEDULL)

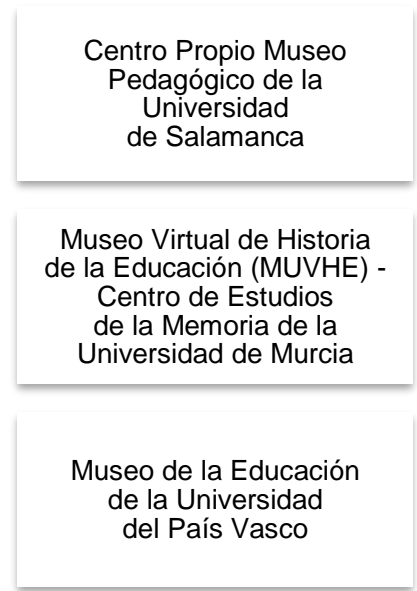

Figura 01 - Museos pedagógicos en España.

Fuente: Cuadro elaborado a partir de ÁLVAREZ, Pablo; PAYÁ, Andrés. Los Museos Pedagógicos en España: Tradición y futuro ante la difusión del Patrimonio Histórico Educativo. In: RIVERA, Roberto; GARCíA FERNÁNDEZ, Isabel (Coords.). Museos y colecciones de la Universidad Complutense de Madrid. Madrid: Universidad Complutense de Madrid, 2015, p. 239-240. 
Como han puesto de manifiesto los profesores Pablo Álvarez y Andrés Payá (2015), los museos pedagógicos universitarios se presentan como un instrumento imprescindible para la reconstrucción histórico-educativa de nuestra memoria escolar. Se conciben como un recurso para la docencia y la investigación, formando parte de los programas de formación de los futuros maestros/as y pedagogos/as de la sociedad del futuro. En este contexto universitario, un museo pedagógico, también denominado Museo de Pedagogía, Enseñanza y Educación por el Comité Internacional de Museo (ICOM), es una ventana abierta al presente y al pasado de la educación, y a la proyección de su futuro. (ÁLVAREZ DOMÍNGUEZ, 2010).

Recientemente se acaba de publicar un libro de gran interés para la museística, coordinado por el profesor Pablo Álvarez (2016), donde se recoge una muestra representativa de diecisiete museos pedagógicos, de los cincuenta que existen en la actualidad en España. En la obra se da cuenta de las funciones y finalidades de todos los museos pedagógicos universitarios distribuidos en gran parte de la geografía española.

En este trabajo, nos vamos a centrar en el Museo Pedagógico de la Universidad Complutense de Madrid, el Museo de Historia de la Educación Manuel Bartolomé Cossío (en adelante Museo MB Cossío) como un ejemplo de espacio dinamizador para la reconstrucción de la memoria escolar colectiva.

\section{El Museo de Historia de la Educación Manuel Bartolomé Cossío de la Universidad Complutense de Madrid}

El Museo MB Cossío, pertenece a la Facultad de Educación de la Universidad Complutense de Madrid. Posee una amplia colección de materiales y tiene como finalidad la investigación, la docencia en el campo de la Historia de la Educación y la organización de actividades museográficas que contribuyan a difundir el patrimonio histórico-educativo a la comunidad educativa y a la sociedad en su conjunto².

El Museo MB Cossío está concebido como un laboratorio de Historia de la Educación. Esta configuración responde a planteamientos pedagógicos que lo articulan como un taller de aprendizaje activo que permiten al usuario conectar con una historia viva que favorezca sentir su necesidad y su sentido. También aspira a ser un laboratorio donde investigar sobre distintos elementos del currículo escolar español en épocas pasadas, lo que incluye, por supuesto, la recuperación del patrimonio histórico escolar, así como la catalogación de documentos escolares diversos. Por otra parte, funciona como un centro de intercomunicación de culturas, ya que su ubicación en una población escolar muy numerosa, le obliga a prepararse para recibir a los diversos centros educativos que deseen iniciar a sus alumnos y alumnas en la recuperación de la memoria histórico-escolar, así como a los particulares interesados, a la vez que muestra a todos y todas las madrileñas, de todas las edades, los elementos de las culturas escolares en que han sido formados 0 que les antecedieron. Y, por supuesto, el objetivo más importante de este museo se dirige

\footnotetext{
${ }^{2}$ Actualmente las profesoras del Departamento de Teoría e Historia de la Educación, Teresa Rabazas y Sara Ramos, en calidad de directora y secretaria respectivamente llevan la gestión del Museo. Asimismo, cuenta con un becario o becaria, en horario de mañana para la atención al público y visitas programadas. El Museo dispone de un espacio (aula 0102) constituido por dos salas en la planta baja de la Facultad de EducaciónCentro de Formación del Profesorado. C/ Rector Royo Villanova, 1. Ciudad Universitaria. 28040 Madrid. Teléfono: 91394 6326. E-mail: museombcossio@edu.ucm.es. Web: https://educacion.ucm.es/museomanuel-bartolome-cossio.
} 
a conformarse como un centro de conservación y análisis del patrimonio histórico-educativo de la Comunidad, que colabora en el rescate del patrimonio histórico-escolar de tal Comunidad de Madrid, en su conservación y en la propagación de su conocimiento a través de sucesivas exposiciones, intentando evitar cualquier sensación de anquilosamiento, rigidez $o$ inutilidad.

El Museo es miembro institucional de la "Sociedad española para el estudio del patrimonio histórico-educativo (Sephe)" desde el año 2004 y sede de la misma. Esta Sociedad tiene como finalidad avanzar en el conocimiento histórico educativo, contribuir al desarrollo de nuevos enfoques museísticos, y colaborar en la promoción de las potencialidades explicativas de la memoria y el patrimonio de la cultura escolar. Todo ello, a través de tres líneas de actuación fundamentales. En primer lugar, la protección y conservación del patrimonio histórico educativo; en segundo lugar, el estudio e investigación del mismo; y por último, la promoción, estímulo, apoyo y difusión de las acciones relacionadas con los fines expuestos anteriormente.

El Museo MB Cossío comenzó su andadura en 1989 de la mano de un grupo de profesores y profesoras de Historia de la Educación que mostraron interés por la recuperación y preservación de la cultura material e inmaterial de la escuela. Dicho Museo a lo largo de sus tres décadas ha contado con la colaboración de diferentes docentes del Departamento de Teoría e Historia de la Educación, ejerciendo su tarea de manera honorífica, voluntaria y altruista, siendo sus principales impulsores, los profesores Julio Ruiz Berrio (Director) y Anastasio Martínez Navarro, Francisco Canes y las profesoras Carmen Colmenar y Miryam Carreño. Paralelamente, se han ido incorporando profesoras más jóvenes, desempeñando diversas funciones y terminando en la dirección del mismo en el momento actual. A lo largo de toda su existencia, también ha sido decisiva la ayuda y colaboración de los estudiantes de Pedagogía para el desarrollo de las propuestas puestas en marcha.

Por otra parte, es necesario situar su origen en un contexto clave en el que las reformas educativas, especialmente la concentración escolar iniciada con la Ley General de Educación de 1970 y la renovación de materiales por el aumento de las disponibilidades presupuestarias, pusieron en peligro gran parte del patrimonio educativo de la provincia y del conjunto del país.

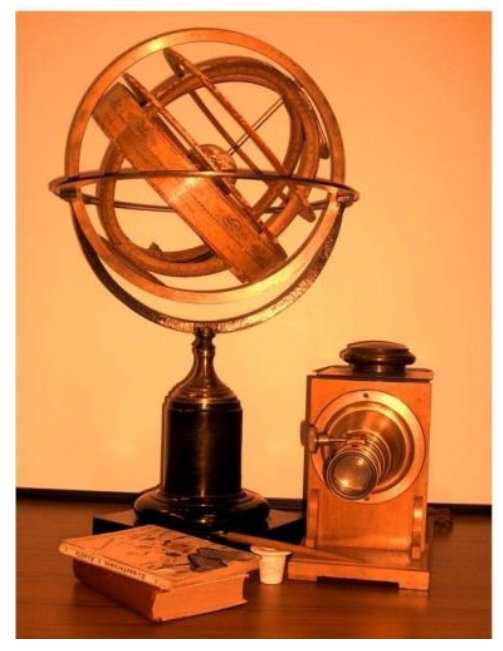

Figura 01 - Logotipo del Museo.

Fuente: Museo MB Cossío. 
Sus primeras acciones se encaminaron a preservar y salvaguardar un fondo documental y material procedente de un colegio público de Madrid. La remodelación del Colegio Nuestra Señora de La Paloma provocó el desmantelamiento del archivo y fondo bibliográfico. Gracias a la preocupación de los profesores Anastasio Martínez Navarro y Julio Ruiz Berrio por la conservación de este patrimonio, se logró establecer un convenio de colaboración con el Ayuntamiento de Madrid en 1990, autorizando a la Facultad de Educación la custodia y salvaguarda del archivo procedente de una institución escolar. El profesor Martínez Navarro (1998, p. 274-275) expone los objetivos que se establecieron en el mencionado convenio:

- Clasificar, catalogar y conservar los documentos y materiales escolares antiguos de las escuelas dependientes de la administración municipal de Madrid;

- Ofrecer la posibilidad a los estudiantes de Historia de la Educación de participar efectivamente en las campañas de recuperación de fondos documentales;

- Ofrecer a los alumnos de las escuelas públicas municipales la ocasión de realizar experiencias prácticas en el marco de las ciencias sociales, gracias a las visitas o a los trabajos en el Museo.

Fruto de este acuerdo, el Ayuntamiento de Madrid trasladó el fondo del Colegio a la Facultad de Educación, constituido por manuales escolares, obras de pedagogía, material didáctico, algo de mobiliario escolar, documentos administrativos, cuadernos escolares, exámenes, etc. Este conjunto patrimonial fue conservado en calidad de depósito en los locales cedidos por la Facultad y a partir de ese momento, se puede considerar que el proyecto museístico comienza a consolidarse con el apoyo institucional del Decanato de la Facultad.

En cuanto a sus características físicas, el Museo cuenta con dos salas permanentes. La primera de ellas (Figura 02), desde la cual se accede al Museo, constituye el espacio principal o lugar de trabajo en el que se desarrollan las actividades de consulta e investigación así como las actividades docentes, principalmente los seminarios de investigación de Historia de la Educación, de ahí que reciba el nombre de "laboratoriotaller", así como las tareas de recuperación y catalogación de los fondos del Museo. En dicho espacio se encuentra gran parte del patrimonio histórico-educativo proveniente del mencionado Colegio.

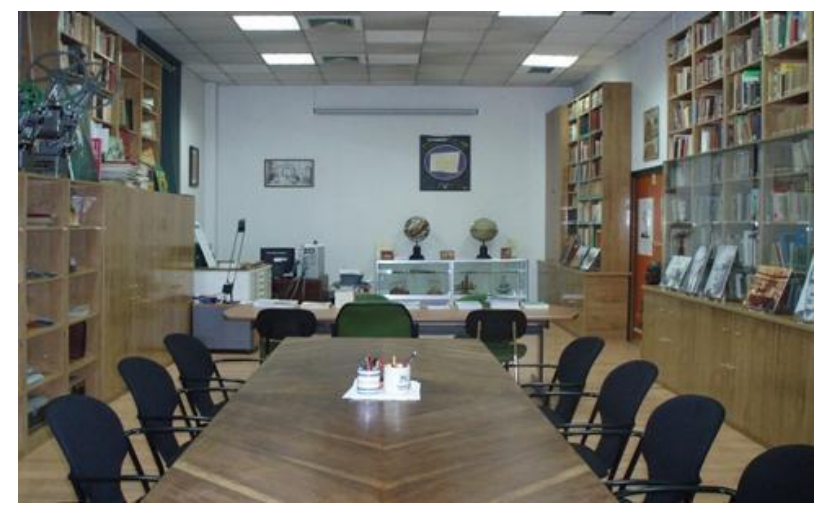

Figura 02 - Sala principal de Museo de Hª de la Educación M. B. Cossío.

Fuente: Museo MB Cossío. 
La segunda sala (Figura 03) muestra una colección de instrumentos y material didáctico del siglo XIX y parte del XX para la enseñanza de las Ciencias Experimentales de gran valor histórico porque proceden de los antiguos laboratorios de las Escuelas de Magisterio, concretamente la Escuela de Magisterio Pablo Montesino - primera Escuela Normal Central de maestros, fundada en 1839 por el pedagogo Pablo Montesino - y la Escuela de Magisterio María Díaz Jiménez - creada en 1858 como Escuela Normal Central de maestras -. La colección se encuentra inventariada y la mayor parte está formada por instrumentos relacionados con la enseñanza de la Física y Biología. También dispone de algunos materiales relacionados con la Química, aunque conviene señalar que al tratarse de un material frágil, fabricado en su mayoría con vidrio, ha resultado muy complicada su conservación. La doble procedencia de los fondos conforma la especificidad de este museo frente a otros, del ámbito estatal como el Museo La escuela de ayer de Huesca, el Museo del Niño y Centro de Documentación histórica de la escuela de Albacete, el Museo escolar de Galicia (Mupega) o el Centro Internacional de Cultura Escolar (Ceince).

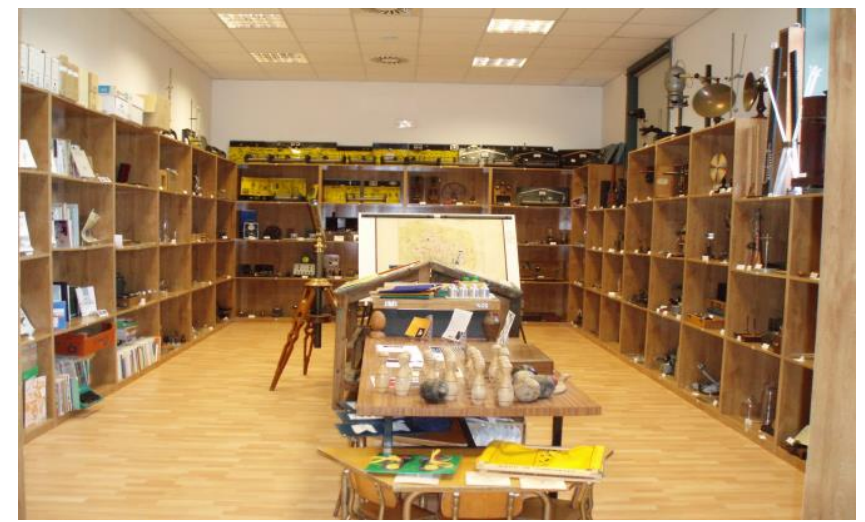

Figura 03 - Sala 2 - Instrumentos de Ciencias Experimentales.

Fuente: Museo MB Cossío.

Por otra parte, las actividades museográficas del Museo se desarrollan en los aledaños del Museo, en la planta baja de la Facultad, en la que encuentra una serie de vitrinas y expositores ubicados en un espacio amplio donde el Museo adquiere un mayor protagonismo, y sus actividades una mayor proyección y difusión entre el alumnado de la Universidad (Figura 04). En palabras de su anterior director, Julio Ruiz Berrio, "el Museo y sus exposiciones no se quedan arrinconados en un almacén sino que salen al encuentro de su posible clientela". (RUIZ BERRIO, 2011).

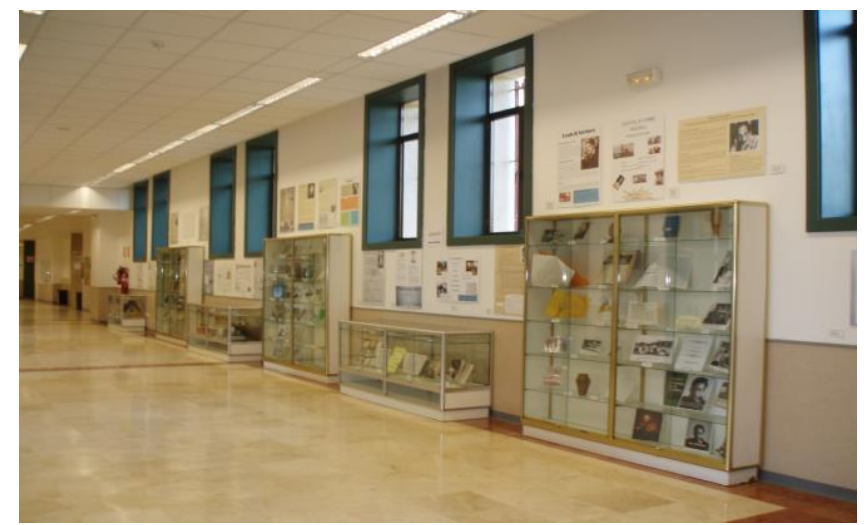

Figura 04 - Sala de exposiciones. Hall de la Facultad de Educación.

Fuente: Museo MB Cossío. 


\section{Las Colecciones del Museo de Historia de la Educación MB Cossío}

Entre las colecciones del Museo MB Cossío, cabe destacar los más de cinco mil volúmenes de manuales escolares del siglo $\mathrm{XX}$, mayoritariamente, y un porcentaje menor del siglo XIX pertenecientes a distintos niveles de enseñanza: infantil, escuela primaria y bachillerato. La procedencia de estos libros es múltiple, predominando las donaciones institucionales y de particulares, habiéndose adquirido algunos de ellos por compra directa en librerías de anticuario. Esta colección de manuales supone una riqueza patrimonial muy interesante para el investigador y el alumnado de Historia de la Educación porque estos textos nos dejan entrever algunos aspectos del jardín oculto del currículo de la escuela: programas, contenidos, metodología, imágenes, ideología, actividades, etc. El giro historiográfico hacia la microhistoria nos permite desvelar los silencios de la Historia de la Educación que señaló Harold Silver hace ya más de dos décadas (1982). El análisis de los manuales como los cuadernos o las imágenes nos acercan a la caja negra de la escuela, nos muestran la escuela por dentro, los métodos que se emplearon en la enseñanza, qué modelos de examen y disciplina se aplicaban, así como otros aspectos que permitan conocer la realidad educativa. Además de los más de 5.000 manuales escolares y libros de lectura (siglos XIX y XX), el Museo conserva en torno a unas 1.525 monografías pedagógicas (s. XX); 313 libros de historia general (s. XX); 582 objetos escolares (linternas mágicas, proyectores, ábacos, etc.); 641 instrumentos científicos completos; 890 memorias de distintas instituciones educativas y su entorno de los años 50 y 60; Colección de revistas (24 m lineales); Documentos educativos (30 m lineales); 60 mapas, entre los que se incluyen ejemplares de Paul Vidal de LaBlache - que podrían ser un legado de D. Ricardo Beltrán y Rózpide, primer catedrático de Geografía en la Escuela Superior de magisterio y en la Sección de Pedagogía de la Universidad de Madrid, y discípulo de aquel- (Rodríguez Esteban, 1997); 893 películas y 400 casetes con entrevistas a personas que fueron a la escuela en los años treinta y cuarenta ${ }^{3}$.
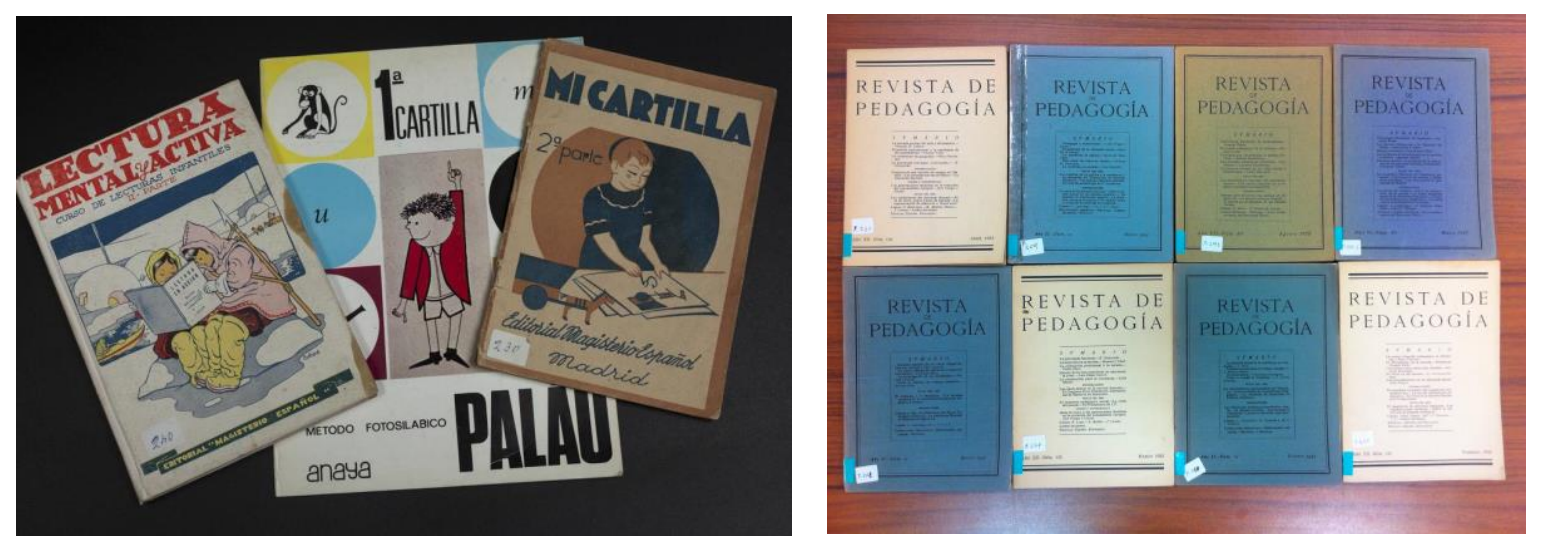

Figura 05 - Cartillas escolares y prensa pedagógica (Revista de Pedagogía).

Fuente: Museo MB Cossío.

\footnotetext{
${ }^{3}$ Durante todos estos años, el Museo como organismo vivo, ha incorporado nuevos fondos procedentes de donaciones particulares e institucionales, entre los que podemos destacar el Fondo Zacarias, perteneciente a la donación realizada por Zacarías Ramo Traver, Inspector de Enseñanza, o el Fondo SIGE, procedente del Seminario Interdisciplinar Género y Educación, compuesto por profesoras de la Facultad que desde distintas áreas de conocimiento investigan sobre educación y género. Asimismo el alumnado realiza donaciones particulares de manera continuada. Recientemente, se ha recibido un donativo amplio del Servicio de Inspección Educativa, de la Dirección del Área Territorial de Madrid.
} 
También se dispone de aparatos representativos del uso del cine en la escuela. El Museo cuenta con 3 máquinas de cine de diverso cortometraje, aunque lo más destacado sea la colección de más de 100 películas que fueron utilizadas en los años 60 y 70 para la didáctica de diferentes materias. Como antecedente del cine el Museo tiene 2 linternas mágicas (Figura 06), de principios del siglo XX, así como una pequeña colección de transparencias grabadas en vidrio.

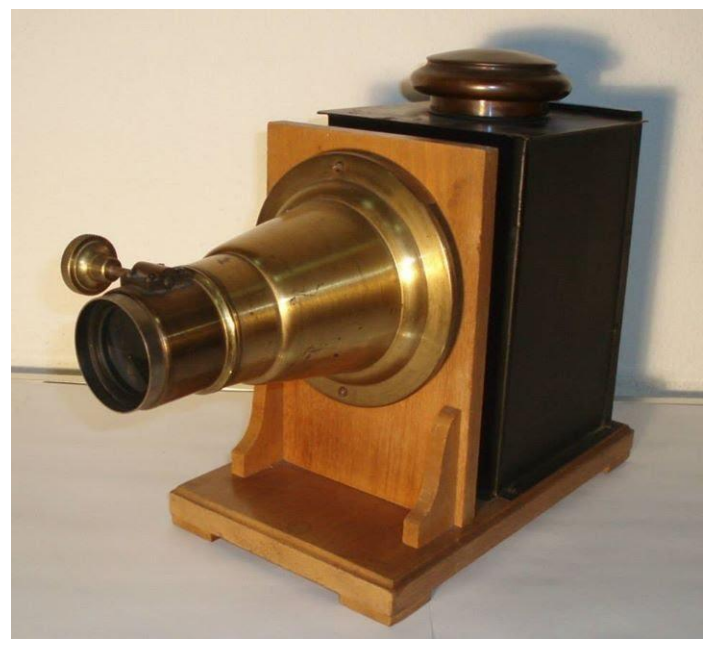

Figura 06 - Linterna mágica.

Fuente: Museo MB Cossío.

Igualmente merece recordar la colección de mapas y de láminas para la enseñanza de la Geografía y la Historia Natural. La mayor parte fueron editadas en la primera mitad del siglo XX, predominando los elaborados por editoriales francesas y españolas. Asimismo, para la didáctica de la Geografía se cuenta también con una esfera armilar y un globo terráqueo (Figura 07) fabricados en el siglo XIX, de gran valor, así como una bola del mundo moderna.
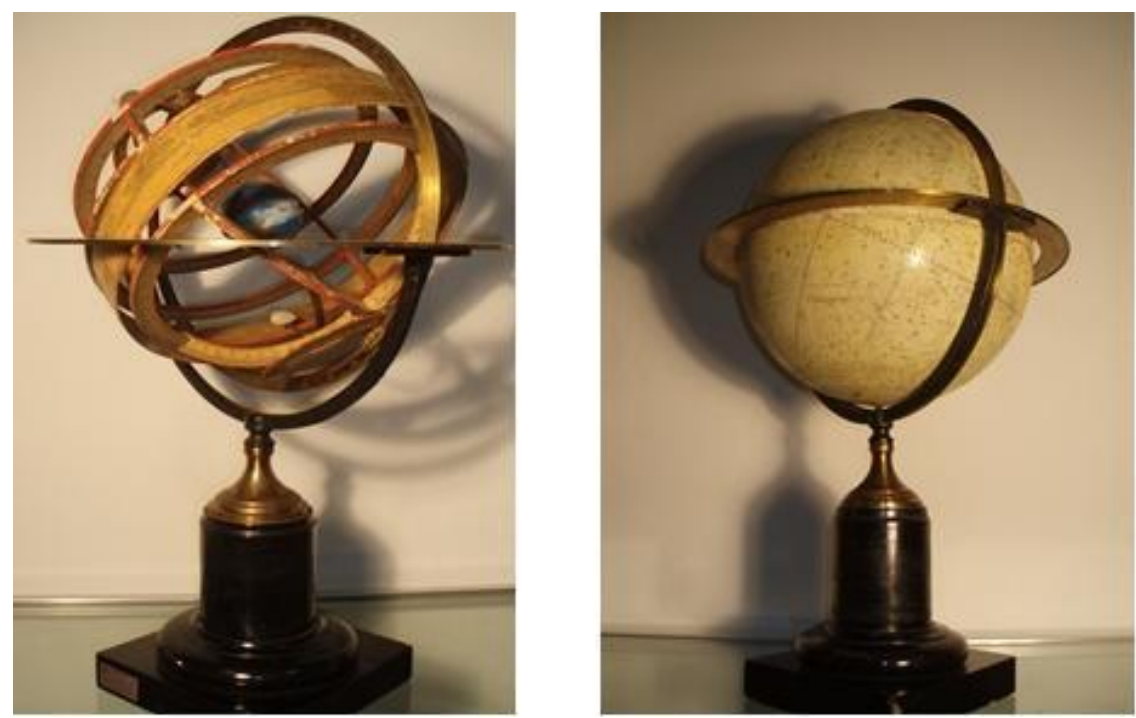

Figura 07 - Esfera armilar y Globo terráqueo.

Fuente: Museo MB Cossío. 
En relación con la evolución de la enseñanza de la escritura, el Museo dispone también de algunos instrumentos interesantes, que podemos recordar por orden cronológico: una pluma de ave como testigo de finales del siglo XVIII, plumines metálicos montados sobre palilleros (mediados s. XIX y primera mitad del s. XX), el pizarrín y la pizarra. Por último, señalar la introducción del bolígrafo en la escuela, a partir de los años 60. El Museo cuenta con diversos útiles para el dibujo artístico o el dibujo técnico: escuadras, reglas, cartabones de madera, compases de madera y tiza, estuches de tinta china, plumines, etc. Aunque reviste especial interés las dos cajas portátiles correspondientes a pequeñas imprentas manuales que dan cuenta de las innovaciones educativas que se aplicaron en las escuelas españolas.

Así también cuenta con materiales de algunos sistemas de enseñanza, entre los cuales destacamos los dones de Fröbel, materiales del método Montessori y cuadernos y objetos pertenecientes a la pedagogía Waldorf. Existen también algunos recursos didácticos para la enseñanza de diversas materias, Lenguaje, Aritmética, Ciencias, etc. Como por ejemplo, los puzzles o juegos didácticos para hacer más atractiva la enseñanza fabricados en los años 30 y 40.

Respecto a la colección de láminas se dispone de una buena muestra sobre artes aplicadas e industriales, y a ella se pueden añadir otras sueltas en torno a las anatomía y fisiología del cuerpo humano, a la Historia Sagrada, y a diferentes motivos, desde grabados o pinturas artísticas hasta varios carteles anunciadores. Carácter más singular tienen una serie de objetos escolares bastante representativos de amplios periodos del siglo XX, de los que quedan muy pocos ejemplares. Entre ellos podríamos citar algunos símbolos religiosos y patrióticos: retratos de Francisco Franco, José Antonio Primo de Rivera, Virgen de la Paloma, banderas nacionales, unas huchas de petición por las Misiones o bien algunos aparatos de reeducación de sordomudos, etc.

Por último, quisiéramos mencionar otra de las colecciones de índole diversa en la temática, pero de gran valor pedagógico y del patrimonio histórico educativo, como es el Fondo Romero Marín, compuesto por un conjunto de Memorias o trabajos de fin de curso de las prácticas de Pedagogía, que realizaron los alumnos durante las décadas de los años 50,60 y principios de los 70 . Cuenta con casi unos 900 trabajos monográficos de pedagogía general o memorias de fin de curso, que realizaron los estudiantes de la Facultad de Filosofía y Letras, pertenecientes a la Sección de Pedagogía entre 1950 y 1975, a petición del profesor Catedrático de Pedagogía General y Racional de dicha Sección Anselmo Romero Marín - nombrado como Catedrático el 22 de marzo de 1949, BOE 8 de abril (RUIZ BERRIO, 2005, p. 139). Los estudiantes procedían de distintos lugares de la geografía española, y se les pedía realizar un trabajo sobre una realidad educativa, en algunos casos vinculada a su actividad laboral, dado que una parte del alumnado ya ejercía profesionalmente en el ámbito educativo (COLMENAR ORZÁES, 2010, p. 348-349). En tal sentido, las memorias ofrecen estudios sobre distintos ámbitos de la enseñanza y diversos niveles educativos, así como de distintas zonas geográficas, tipos de población estudiantil, y de temáticas complejas como el analfabetismo, la pobreza, la educación especial, la educación de las mujeres, la enseñanza privada, la enseñanza pública, las escuelas rurales, las escuelas urbanas, la formación profesional, etc. Este patrimonio históricoeducativo se amplía con una variada y rica documentación educativa que va desde estadísticas, datos geográficos, pruebas diagnósticas hasta un fondo fotográfico que abre 
una ventana privilegiada a la práctica educativa de aquellos años. Por todo ello, se puede afirmar que el Fondo Romero Marín (en adelante FRM) posee un gran valor etnográfico. En estos momentos se está realizando un archivo fotográfico a disposición de la comunidad universitaria en un entorno virtual. Los miembros del Grupo de Investigación Consolidado Historia y presente de la cultura escolar: género e identidades (en adelante utilizaremos el acrónimo Cegi) ${ }^{4}$ han comenzado ya a explorar sus posibilidades historiográficas y los primeros resultados han sido presentados en el III Foro lbérico de Museismo Pedagógico y VI Jornadas Científicas de la Sociedad Española para el estudio del Patrimonio Históricoeducativo (Sephe) ${ }^{5}$.

\section{El Museo de Historia de la Educación MB Cossío como Plataforma de Aprendizaje}

Desde sus orígenes como Museo Universitario de Historia de la Educación, sus diversas actividades han tenido como finalidad la investigación y la docencia evolucionando al ritmo de los tiempos y a las demandas académicas y socioeducativas. En los primeros años de andadura (1990-1999), se centró en la puesta en marcha de seminarios teóricoprácticos de investigación histórico-educativa como actividades complementarias y voluntarias a las clases de Historia de la Educación al objeto de sensibilizar y motivar al alumnado para iniciar trabajos de investigación en esta área de conocimiento. Ya el mismo proceso de levantado de inventario in situ, de selección y catalogación del fondo almacenado en el Colegio de Nuestra Señora de La Paloma, conformó una de sus primeras actividades prácticas que a través de un seminario sobre documentación y catalogación bibliográfica, introduciría a los participantes en la tarea heurística. La segunda actividad se centró en el entorno educativo del que procedían los fondos y la tercera tuvo como foco de estudio la escuela en el Franquismo. Para ello, se usó como fuentes documentales las memorias del Fondo Romero Marín que, sobre distintos centros e instituciones educativas y asistenciales coetáneas, redactaron los estudiantes en los años 50 y 60 . (MARTíNEZ NAVARRO, 1997).

En un segundo periodo (2000-2010), los seminarios llevados a cabo por los profesores/as Julio Ruiz Berrio, Teresa Rabazas Romero y Sara Ramos Zamora durante los años 2003, 2004 y 2005 sobre Catalogación y documentación museística. Introducción a la investigación histórico-educativa, o sobre Los manuales escolares como fuente de conocimiento para la cultura escolar y la Historia de la Educación fueron contribuyendo a esa labor de acercamiento del alumnado de Pedagogía a las actividades de investigación histórico-educativa.

Pero sin duda, las actividades museográficas del Museo MB Cossío constituyen desde su existencia una de las líneas prioritarias con las que se ha pretendido difundir el patrimonio histórico-educativo que alberga. Siempre con un talante cooperativo, en tanto que muchas de las iniciativas han sido llevadas a cabo en colaboración con entidades relevantes del ámbito educativo como Anele (Asociación Nacional de Editores de Libros y material de Enseñanza), el Sige (Seminario Interdisciplinar Género y Educación), otras Universidades o Bibliotecas Históricas, etc. Las temáticas objeto de las exposiciones han

4 En su página web se puede encontrar más información: <www.pixelcpg.com/clientes/grupo_invest/ cultura_escolar.html>.

5 Véase en el apartado de referencias bibliográficas los trabajos publicados hasta la fecha. 
ido al compás de la evolución de la historiografía educativa emergente en cada momento pero también se han programado como actividades vinculadas a actos conmemorativos culturales, a congresos pedagógicos y especialmente, en la etapa más actual del Museo, a la docencia vinculada a la Historia de la Educación -de la que hablaremos más adelante-. Estas últimas, se han desarrollado siempre en colaboración directa con los estudiantes, de manera que ellos y ellas han pasado a un primer plano al ser agentes principales en la planificación y desarrollo de las mismas. En tal sentido, el Museo ha puesto en marcha exposiciones vinculadas a los manuales escolares; a la Historia de la Educación de las mujeres; a la Historia de la renovación pedagógica, etc. ${ }^{6}$ Todas estas iniciativas, no han tenido otro objeto que el apuntado por su primer director del Museo, Julio Ruiz Berrio:

Un Museo de Historia de la Educación es claramente un establecimiento con profesores de Historia de la Educación y profesores expertos en todas las especialidades didácticas, con alumnos que pueden recibir clases prácticas, seminarios y cursillos sobre el terreno o que en su momento pueden realizar investigaciones específicas sobre parte del material conservado. Por otro lado, está abierto a los colegios que deseen mostrar a sus alumnos la memoria históricoescolar de su tierra y de sus gentes, así como a los particulares interesados. Y por supuesto, un Museo que rescatará el patrimonio histórico-escolar de la Comunidad de Madrid, lo conservará y lo dará a conocer a través de sucesivas exposiciones, intentando evitar cualquier sensación de anquilosamiento, rigidez o inutilidad." (RUIZ BERRIO, 2008, p. 8-9).

Por tanto, el Museo ante todo, fiel a su origen, mantiene su vocación de laboratorio de investigación histórica que le conforma como una herramienta didáctica para las asignaturas relacionadas con la Historia de la Educación, organizando prácticas docentes para las asignaturas del Grado y Postgrado que tiene el profesorado de Historia de la

\footnotetext{
${ }^{6}$ Se pueden destacar entre otras, de la primera etapa, la exposición de 1995 bajo el título El hombre y la tierra en la escuela madrileña de principios de siglo. Un año más tarde, en 1996 se organizó la exposición En torno a Pestalozzi en conmemoración del 250 aniversario del nacimiento del pedagogo suizo, vinculado a un Coloquio Internacional sobre La recepción de la Pedagogía pestalozziana en las sociedades latinas celebrado en Madrid. De su segunda etapa, cabe destacar en el año 2000 la exposición El libro y la educación. De las Cortes de Cádiz a la LOGSE con motivo del XXII Congreso Internacional de Historia de la Educación. También en esta etapa desde el año 2001 al 2003 se llevó a cabo la exposición Leer y Escribir en Madrid en el siglo XX. Asimismo, en colaboración con Anele y vinculado a un Seminario que la Asociación celebró en la Facultad de Educación se expuso la actividad museográfica titulada Los libros escolares y la lectura ante la Loce. En el año 2004 con motivo del Primer Centenario de la creación de la cátedra de Pedagogía Superior en la UCM, el Museo organizó la exposición La pedagogía Científica en la UCM en colaboración con la Biblioteca Histórica de Marqués de Valdecilla de la UCM.

A partir del año 2005, la exposición llevada titulada La educación de las niñas en la España contemporánea (1875-1975) y en el año 2009, en colaboración con el Sige, Educar a las mujeres. España, 1875-1975, contribuyeron a consolidar los estudios de género, una de las líneas de investigación más desarrolladas entre el profesorado de Historia de la Educación.

En la etapa más actual, durante los cursos académicos 2012-2013, 2013-2014, 2015-2016, vinculadas a la asignatura Historia y Corrientes Internacionales de la Educación, se llevaron a cabo exposiciones docentes sobre Pasado y presente de la Renovación Pedagógica o la exposición Pasado y Presente de las prácticas de Pedagogía. Más recientemente en el año 2016 el Museo se ha visibilizado a través de exposiciones como la realizada en la Semana de las Letras Viaja a un mundo lleno de imaginación en un abrir y cerrar de hojas; o en el Día de los Museos, con la exposición Hay una cita para ti... en el MUSEO; en la Semana de la Ciencia con la exposición Museo, educación y ciencia: un espacio compartido; en diciembre de 2016 con la exposición Érase una vez...la Navidad en el siglo XX: cuentos y juguetes. Vinculada al XVI Congreso Nacional y VII Iberoamericano de Pedagogía: Democracia y Educación en el Siglo XXI, celebrado en la Facultad de Educación, el Museo MB Cossío contribuyó con una exposición titulada Democracia y Educación en el siglo XXI. La obra de John Dewey 100 años después. Recientemente conmemorando el Día Internacional de la Mujer, y en colaboración con el Sige en marzo de 2017 llevó a cabo la exposición La mujer, esa gran desconocida.
}

Hist. Educ. (Online) Porto Alegre v. 21 ก. 53 set./dez. 2017 p. $100-119$ 
Educación: Historia y Corrientes Internacionales de la educación y la Cultura; Historia del pensamiento pedagógico; Historia de la educación de las mujeres; Máster de Investigación en Educación; Máster de Investigación en Estudios Feministas; Máster Interuniversitario Memoria y Crítica de la educación o el Máster Interuniversitario El Patrimonio Cultural en el siglo XXI: Gestión e investigación, entre otros.

En tal sentido, se ha formalizado y cotidianizado en la docencia el uso didáctico del patrimonio histórico-educativo del Museo, favoreciendo la reconstrucción de la historia de la escuela desde otra mirada pedagógica, más etnográfica a través de su cultura material e inmaterial. Su uso, está permitiendo a estudiantes de grado en Pedagogía, Magisterio de Educación Primaria e Infantil y grado de Educación Social, construir "posibles comunidades interpretativas de historiadores desde la base del análisis intersubjetivo de los lenguajes que portan estos materiales o que suscitan sus lecturas semiológicas" (ESCOLANO, 2009, p. 7). Dicho de otro modo, este Museo representa un entorno clave para las investigaciones de los estudiantes, como laboratorio patrimonial de la educación fomentando un aprendizaje activo, siendo capaces de propiciar el contexto más idóneo para iniciar al alumnado en la investigación histórico-educativa. Además, resulta ser un recurso didáctico para "educar en el patrimonio histórico-educativo y en la educación patrimonial, promoviendo el conocimiento, comprensión, respeto y cuidado de dicho patrimonio" (LÓPEZ; BERNAL, 2009, p. 55) y en última instancia, para crear una sensibilidad patrimonial con la ciudadanía. (PADRÓs; COLELLDEMONT, 2014, p. 109).

Teniendo presente la naturaleza y orígenes del Museo Pedagógico Nacional de 1882 dirigido por Manuel Bartolomé Cossío, este museo universitario pretende ser también una agencia de modernización pedagógica. A través de la docencia en las asignaturas de Historia de la Educación los estudiantes han hecho uso de su patrimonio para reconstruir la historia de la renovación pedagógica a lo largo de historia. Para el estudio de los autores y autoras vinculados a la historia de la renovación pedagógica, se llevan a cabo periódicamente sesiones prácticas en las que los estudiantes consultan de forma directa fuentes primarias, como objetos pertenecientes a la cultura material de la escuela, manuales escolares así como la prensa pedagógica. El uso de estas fuentes documentales ha fomentado la capacidad de inferir conclusiones, interpretar y correlacionar conceptos, siendo además, "un reto importante en el planteamiento científico de la enseñanza y el aprendizaje de la historia" (FELIU; HERNÁNDEZ, 2011, p. 10). Resulta de especial importancia la consulta de la Revista de Pedagogía7, por ser el órgano de difusión en España del Movimiento de Escuela Nueva y por ser la revista que leían maestros, directores, profesores de instituto y universitarios en el primer tercio del siglo XX. Es una revista que aportó informaciones muy valiosas sobre la "renovación, reforma y mejora de la escuela difundiendo las innovaciones metodológicas más relevantes de la pedagogía vinculada al movimiento internacional de Escuela Nueva" (LÓPEZ; DELGADO, 2014, p. 75). Además el manejo de esta riqueza documental y material perteneciente a los métodos y técnicas de los autores vinculados a las distintas corrientes educativas, ha favorecido la adquisición de competencias en la extracción de información, pues "se revela como fundamental en el proceso de formación con respecto a la historia [de la educación]. Esta

\footnotetext{
7 RAMOS ZAMORA, S. y PERICACHO GÓMEZ, F. J. (2015). Una propuesta de innovación docente para enseñar historia de la renovación pedagógica. Educaciò i Història. Revista d'Història de l'Educació, 26, p. 65-88.
} 
capacidad va a tener infinitas posibilidades de reaplicación en la vida cotidiana y en una perspectiva crítica de optimización de la persona y de su proyección cívica". (FELIU; HERNÁNDEZ, 2011, p. 14).

En la actualidad el Museo MB Cossío forma parte de la Red de Museos de la Universidad Complutense de Madrid apoyados institucionalmente por la Unidad de Gestión del Patrimonio Histórico (UGPH), organismo creado para la gestión de bienes muebles del Patrimonio Histórico y su conservación así como el asesoramiento sobre los aspectos museológicos, la realización de exposiciones y la promoción y difusión de todo tipo de actividades culturales relacionadas con el patrimonio artístico, científico o técnico. En ese marco, otra de sus líneas prioritarias de actuación se enmarcan en la dinamización de proyectos de investigación e innovación docente, al ser centro neurálgico de investigaciones por parte de profesorado de Historia de la Educación, que conforma el Grupo de Investigación Consolidado Historia y Presente de la Cultura Escolar: Género e identidades, vinculado al Campus de Excelencia Internacional de la UCM y englobado dentro del Cluster de Patrimonio Cultural8. En esa dirección, apuntan las colaboraciones con otras instituciones y redes, especialmente museos de educación nacionales e internacionales y otros grupos de investigación ${ }^{9}$, destacando no sólo la Sephe, sede oficial de la misma - como adelantábamos más arriba -, la Ridhpe (Red Iberoamericana para la Investigación y Difusión del Patrimônio Histórico Educativo) o la Red de los Institutos históricos, entre otros y otras. Asimismo a través de su patrimonio experimental está abriéndose a los especialistas de las didácticas de las ciencias para que pongan en valor la impresionante colección de instrumentos científicos.

El énfasis manifestado en la oralidad y en la materialidad de la nueva museología y de la Nueva Historia Social de la Educación ha favorecido que el Museo se haya interesado en colaborar con la Universidad de Mayores en las tareas de recuperación de la memoria histórica a través de la memoria oral de sus alumnos y alumnas así como la recuperación del material docente del profesorado universitario de la Facultad de Educación a través de sus apuntes de clase, de preparación de lecciones, etc. para contribuir a un mayor conocimiento e historización de la propia Facultad.

En su dimensión socioeducativa, el Museo está llevando a cabo varias iniciativas en la actualidad. En estos momentos, está siendo pionero como museo universitario en la puesta en marcha de una iniciativa clave para fomentar la educación inclusiva e integradora de personas con diversidad funcional. En estos momentos ha iniciado un proyecto de integración sociolaboral, desarrollado en colaboración con la Asociación Pauta, dedicado a la intervención educativa de personas con autismo. Actualmente está colaborando un joven con autismo de la Asociación que viene realizando durante el curso académico 2016-2017

\footnotetext{
${ }^{8}$ Se pueden destacar el proyecto de investigación: "Creación de un museo virtual de etnografía escolar de la España contemporánea”, dirigido por el profesor D. Julio Ruiz Berrio, perteneciente al Plan Nacional de Investigación Científica, Desarrollo e Innovación Tecnológica $(I+D+I)$. CICYT. Ministerio de Educación y Ciencia (2005-2008) (referencia: SEJ2005-07116/EDUC). “Género y Museografía de la Educación” dirigido por la profesora D ${ }^{\mathbf{a}}$ Miryam Carreño Rivero financiado por la Universidad Complutense de Madrid, ayuda competitiva asociada a los Grupos consolidados de Investigación de la UCM concedida al Grupo de Investigación consolidado "Historia y Presente de la cultura escolar. Género e identidades" (2008 a 2012) (referencias: 941322; 941322-853 y CCGO7-UCM/HUM-2280.)

${ }^{9}$ La difusión del alcance pedagógico y social del Museo de Historia de la Educación Manuel Bartolomé Cossío fue presentado por las profesoras Teresa Rabazas y Sara Ramos al Congreso Internacional de Museos Universitarios. Tradición y Futuro celebrado del 3 al 5 de diciembre de 2014.
} 
prácticas acompañadas de un Preparador Laboral y de la Becaria del Museo, consistente en la realización de actividades de merchandising del Museo, así como la participación en las visitas guiadas. El plan de integración trazado conjuntamente con la Asociación Pauta, está favoreciendo que desde el ámbito universitario y desde una Facultad de Educación, se estén rompiendo barreras y desmontando los mitos sobre las dificultades que tienen los autistas para relacionarse con los demás. En esta experiencia por contra, se están logrando resultados muy significativos no sólo en cuanto a formación laboral, sino también en torno a adquisición de competencias sociales muy determinantes.

\section{A modo de conclusión}

En definitiva, los museos universitarios pedagógicos constituyen espacios que permiten reconstruir la memoria educativa colectiva a través del patrimonio históricoeducativo, ya sea material o intangible. Produciéndose un diálogo crítico con la realidad educativa, confrontando la modernidad con el tiempo histórico en el que fueron creados y utilizados. Además, como se ha podido comprobar a lo largo de las tres largas décadas de vida del Museo MB Cossío, se convierten en los mejores escenarios de aprendizaje y agentes dinamizadores activos para el profesorado, estudiantes, investigadores, visitantes de todas las edades y colectivos de diversidad funcional. El Museo MB Cossío quiere contribuir con esta trascendental tarea.

\section{Referencias}

ÁLVAREZ DOMínGUEZ, Pablo (Coord.). Los Museos Pedagógicos en España. Entre la memoria y la creatividad. Trea-Universidad de Sevilla: Gijón-Sevilla, 2016.

ÁLVAREZ DOMíNGUEZ, Pablo. Nuevo concepto de los Museos de Educación. In: RUIZ BERRIO, Julio (Ed.). El patrimonio histórico-educativo. Su conservación y estudio. Madrid: Biblioteca Nueva, 2010, p. 139-167.

ÁLVAREZ DOMínGUEZ, Pablo; PAYÁ RICO, Andrés. Los Museos Pedagógicos en España: Tradición y futuro ante la difusión del Patrimonio Histórico Educativo. In: RIVERA, Roberto; GARCÍA FERNÁNDEZ, Isabel (Coords.). Museos y colecciones de la Universidad Complutense de Madrid. Madrid: Universidad Complutense de Madrid, 2015, p. 237-242.

BLACKWELL HISTORY OF EDUCATION RESEARCH COLLECTION, History of Education Museums and Collections Directory. Northern Illinois University: Dekalb, 1992.

CARREÑO RIVERO, Miryam. Museología y museografía de la educación. In: ESCOLANO BENITO, Agustín. La cultura material de la escuela. En el Centenario de la Junta para Ampliación de Estudios, 1907-2007. Berlanga de Duero: CEINCE, 2007, p. 91-110.

COLMENAR ORZAES, Carmen. El Fondo Romero Marín del Museo de Historia de la Educación "Manuel Bartolomé Cossío". Memorias sobre las prácticas escolares: la educación infantil. In: MORENO MARTÍNEZ, Pedro Luis; SEBASTIÁN VICENTE, Ana (Eds.). Patrimonio y Etnografía de la escuela en España y Portugal durante el siglo XX. Murcia: CEME, SEPHE, 2012, p. 197-210.

. El Museo de Historia de la Educación "Manuel Bartolomé Cossío". In: RUIZ BERRIO, Julio (Ed.). El patrimonio histórico-educativo. Su conservación y estudio. Madrid: Biblioteca Nueva, 2010, p. 339-361.

COSSETO, Milena. II Museo della Scuola-Schulmusesum della Città di Bozano. Turris 
Babel, n. 2, p. 4, nov. 2002.

ESCOLANO BENITO, Agustín. El patrimonio histórico-educativo de la Escuela y de la Historia de la Educación. Cuadernos de Historia de la Educación "El patrimonio históricoeducativo y la enseñanza de la historia de la educación”, n. 6, p. 7-9, 2009.

FELIU TORRUELLA, María; HERNÁNDEZ CARDONA, F. Xavier. 10 Claves para enseñar historia. Barcelona: Graó, 2011.

HERNÁNDEZ HERNÁNDEZ, Francisca. Planteamientos teóricos de la museología. Gijón: Trea, 2006.

LÓPEZ MARTíNEZ, José Damián; BERNAL MARTÍNEZ, Mariano. El material de enseñanza como recurso didáctico en la historia de la educación. Cuadernos de Historia de la Educación "El patrimonio histórico-educativo y la enseñanza de la historia de la educación", n. 6, p. 53-92, 2009.

LÓPEZ MARTÍNEZ, José Damián; DELGADO MARTÍNEZ, Má Ángeles. La enseñanza de las ciencias escolares en la Revista de Pedagogía (1922-1936). Educació i Història Revista d'Història de l'Educació, 24, p. 69-101, citado en p. 75, jul./des. 2014.

MARTÍNEZ NAVARRO, Anastasio. Le Musée d'Histoire de l'Éducation de l'Université Complutense de Madrid. Histoire de l'éducation, n. 178, p. 274-280, 1998.

. Un seminario sobre fuentes históricas en el Museo de Historia de la Educación de la Facultad de Educación de la Universidad Complutense. Revista Complutense de educación, v. 8, n. 1, p. 305-320, 1997.

PADRÓS, Núria; COLELLDEMONT, Eulàlia. Investigar sobre la historia del centro a través del patrimonio educativo. Orientaciones para la práctica. Formar la sensibilidad patrimonial a través de la práctica experimental. In: BADANELLI RUBIO, Ana Mãa; POVEDA SANZ, María; RODRÍGUEZ GUERRERO, Carmen. Pedagogía Museística. Prácticas, usos didácticos e investigación del patrimonio educativo. Madrid: UCM, 2014, p. 109-118.

RABAZAS ROMERO, Teresa; POVEDA, María. El Fondo Romero Marín del Museo "Manuel Bartolomé Cossío". Análisis de las memorias de las prácticas de Pedagogía. In: MORENO MARTÍNEZ, Pedro Luis; SEBASTIÁN VICENTE, Ana (Eds.). Patrimonio y Etnografía de la escuela en España y Portugal durante el siglo XX. Murcia: CEME, SEPHE, 2012, p. 323-336.

RABAZAS ROMERO, Teresa; RAMOS ZAMORA, Sara. El laboratorio/Museo de Historia de la Educación "Manuel Bartolomé Cossío" de la Facultad de Educación de la Universidad Complutense de Madrid. Estado de las colecciones y líneas de actuación. In: MENEZES, Maㅡ Cristina (Org.). Desafios Iberoamericanos: O Patrimônio HistóricoEducativo em Rede. CIVILIS/UNICAMP-RIDPHE-CME/USP. Campinas - São Paulo (Brasil), 2016, p. 229-256.

. El Museo de Historia de la Educación "Manuel Bartolomé Cossío" de la Facultad de Educación de la Universidad Complutense de Madrid. In: SAN ANDRÉS MOYA, M. (Dir.). Museos y colecciones de la Universidad Complutense de Madrid. Madrid: Universidad Complutense de Madrid, 2015, p. 173-179.

. Museografía y docencia en el Museo/Laboratorio de Historia de la Educación Manuel Bartolomé Cossío. In: ÁLVAREZ DOMínGUEZ, Pablo (Coord.). Los Museos Pedagógicos en España. Entre la memoria y la creatividad. Gijón-Sevilla: Ediciones TreaUniversidad de Sevilla, 2016, p. 163-174.

RAMOS ZAMORA, Sara. Labor pedagógica de los Hogares de Auxilio Social a través de las memorias del Fondo "Romero Marín" del Museo de Historia de la Educación "Manuel 
Bartolomé Cossío". In: MORENO MARTÍNEZ, Pedro Luis; SEBASTIÁN VICENTE, Ana (eds.). Patrimonio y Etnografía de la escuela en España y Portugal durante el siglo XX. Murcia: CEME, SEPHE, 2012, p. 337-354.

RAMOS ZAMORA, Sara; PERICACHO GÓMEZ, F. J. Una propuesta de innovación docente para enseñar historia de la renovación pedagógica. Educaciò i Història - Revista d'Història de l'Educació, n. 26, p. 65-88, 2015.

RODRÍGUEZ ESTEBAN, José Antonio. La Geografía en la Escuela Superior del Magisterio (1909-1932). Ería - Revista cuatrimestral de geografía, n. 42, p. 89-106, 1997.

RUIZ BERRIO, Julio. El museismo pedagógico en el mundo: pasado, presente y perspectivas de futuro. In: PEÑA SAAVEDRA, Vicente (Coord.). I Foro Ibérico de Museísmo pedagógico en España e Portugal: itinerarios, experiencias e perspectivas. Santiago de Compostela: Mupega, Consellería de Educación e Ordenación Universitaria da Xunta de Galicia, 2003.

El Museo de Historia de la Educación, Manuel Bartolomé Cossío. In: LOURO FELGUERAS, Margarida (Org.). Inventariando a Escola. Gondomar: Cámara municipal de Gondomar/Faculdade de Psicología e de Ciencias da Educaçao da Universidade do Porto, 2008, p. 8-9.

El patrimonio histórico-educativo. Su conservación y estudio. Madrid: Biblioteca Nueva, 2010.

. Presentación del Museo-Laboratorio de Historia de la Educación Manuel Bartolomé Cossío, 2011. Texto inédito.

. Historia y museología de la educación. Despegue y reconversión de los museos pedagógicos. Historia de la Educación, 25, p. 271-290, 2006.

Manuel Bartolomé Cossío y los comienzos de los estudios de Pedagogía en la Universidad Complutense de Madrid. In: RUIZ BERRIO, Julio (Ed.). Pedagogía y educación ante el siglo XXI. Madrid: Departamento de Teoría e Historia de la EducaciónUCM, 2005, p. 117-142.

SAN ANDRÉS MOYA, Margarita (Dir.). Museos y colecciones de la Universidad Complutense de Madrid. Madrid: Universidad Complutense de Madrid, 2015.

SILVER, Harold. Knowing and not knowing in the history of education. History of Education, v. XXI, n. b1, p. 97-108, 1982.

VIÑAO FRAGO, Antonio. Memoria, patrimonio y educación. Educatio siglo XXI. v. 28, n. 2, p. 17-42, 2010.

TERESA RABAZAS ROMERO es Profesora Titular de Universidad de la Facultad de Educación, perteneciente a la Universidad Complutense de Madrid y Directora del Museo de Historia de la Educación Manuel Bartolomé Cossío.

Dirección: C/ Rector Royo Villanova, s/n. - 28040 - Madrid - España.

E-mail: rabarom@ucm.es

SARA RAMOS ZAMORA es Profesora Contratada Doctora de la Facultad de Educación, perteneciente a la Universidad Complutense de Madrid y Secretaria del Museo de Historia 
de la Educación Manuel Bartolomé Cossío.

Dirección: C/ Rector Royo Villanova, s/n. - 28040 - Madrid - España.

E-mail: sramosz@ucm.es

Recebido em 29 de março de 2017.

Aceito em 02 de maio de 2017. 